\title{
Simulating Metal Distributions in the ICM
}

\author{
C.M. Cress \\ Univ. of Natal, South Africa
}

\begin{abstract}
The metal enrichment of the intra-cluster medium within a hierarchical structure formation scenario is investigated using a combination of $\mathrm{N}$-body + Hydro simulations and semi-analytic models of galaxy formation. In our simulation, rates and sites of star formation are identified and gas particles in the environment of these 'galaxies' are then enriched with metals using various prescriptions. We investigate the paths of enriched particles, demonstrating how gas enriched at higher redshifts is distributed in a cluster after it has formed. We are then able to predict a number of observable quantities such as the radial profiles of metals from both SNII and SNIa and the evolution of metal content with redshift.
\end{abstract}

\section{Introduction}

The theory that dark matter is cold (non-relativistic at the time of decoupling) is well-supported by observations of fluctuations in the Cosmic Microwave Background and by large-scale structure studies. In a CDM-dominated universe structure is built up hierarchically, with smaller structures forming first. By modelling galaxy formation within underlying dark-matter structures, one can obtain insight into how, in these models, material must be recycled in order to reproduce observations. Here, we investigate the radial distribution of heavy elements in the ICM predicted by galaxy formation models based on CDM and note how X-ray observations can be used to constrain models.

\section{Method}

We ran an $\mathrm{N}-$ body + Hydro $(\mathrm{P} 3 \mathrm{M}+\mathrm{SPH}) \tau \mathrm{CDM}$ simulation of a $(64 M p c)^{3}$ box from $\mathrm{z}=30$ to $\mathrm{z}=0$, saving records of particle positions at 30 timesteps. The simulation was constrained to produce a $10^{15} M_{\odot}$ cluster at its centre. Each gas particle had mass $8.7 \times 10^{8} M_{\odot}$ so we were not able to resolve star formation at all and semi-analytic modelling of galaxy formation was used (Kauffmann et al). Galaxy properties associated with each halo were tracked through time and rates and sites of star-formation were identified.

We associated metal production with star formation, allowing for immediate enrichment from Type II supernovae and delayed enrichment from Type Ia supernovae. These metals were then distributed evenly to gas particles within a specified 'distribution radius' $\left(R_{\text {dist }}\right)$ of the centre of the dark matter halo. We 
kept track of the metal content of each gas particle over time. This provides a coarse way of simulating the distribution of metals by winds blown from star formation regions. Metal distributions for specific choices of the delay time and $R_{\text {dist }}$, were tracked over time.

\section{Results}

Some results are given in Figure 1. See Cress for results on inhomogeneities in the metallicity and on the comparison between different clusters in the simulation.
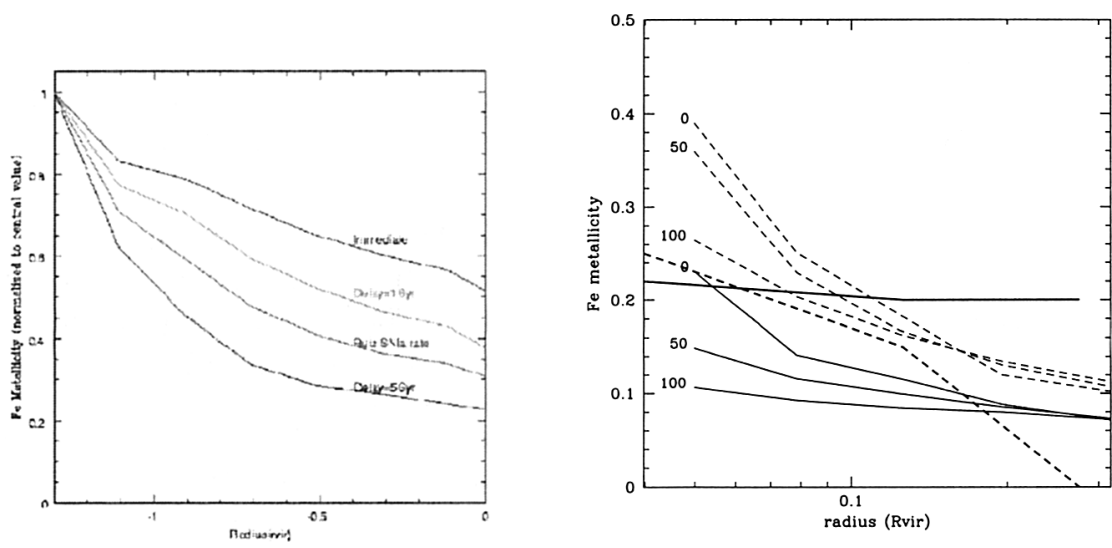

Figure 1. The left figure shows the radial Fe metallicity distribution as a fraction of the virial radius in the central cluster at $\mathrm{z}=0$ for enrichment with varying time delays. The longer the time delay between star formation and metal ejection in galaxies, the steeper the radial profile.We thus expect SNIa elements to have steeper profiles. The right figure shows a comparison of metallicity profile observations as in Finoguenov et al (thick lines) with predictions in models with varying $R_{\text {dist }}$ (given at left of curve in $\mathrm{kpc}$ ): solid lines for immediate enrichment, dotted lines for delayed enrichment. The overall normalisation of the curves is not well determined in the models and curves are offset for comparison. The flat profile observed for SNII elements indicates that distribution radii larger than $100 \mathrm{kpc}$ are required

\section{References}

Cress, C. 2002 in Dark matter in astro- and particle physics. Springer, 90

Finoguenov, A., David, L.P., Ponman, T.J. 2000, ApJ, 544, 188

Kauffmann, G., Colberg, J., Diaferio, A., White, S.. 1999, MNRAS, 307, 529 